\title{
E-Learning
}

Christina Schabasser* und Bert Bredeweg

\section{Ein konzeptionelles Modell des Online-Lernens}

https://doi.org/10.1515/iwp-2019-2027

\section{Intention und Einführung}

Vor dem Hintergrund, dass es sich bei Lehr- und Lernprozessen um dynamische, hochkomplexe Prozesse handelt, erzeugt die Nutzung des Computers und des Internets eine neue Dimension der Komplexität. Inmitten dieser OnlineLehr-Lernumgebungen sind die Lehrenden dazu aufgefordert, Online-Kurse so zu gestalten, dass die Lernenden relevantes Wissen und die damit verbundenen Argumentations- und Problemlösungsstrategien entwickeln können. Der Lernerfolg ist letztlich ein Zusammenspiel verschiedenster Faktoren. Neben einer Vielzahl an anderen Komponenten wirkt sich beispielsweise die Interaktion zwischen Lehrendem und Lernendem äußerst intensiv auf den Lernerfolg aus. Mittels der Erstellung konzeptueller Modelle soll die Komplexität von Online-Lehr-Lernprozessen durch Abstraktion aufgebrochen werden. Erst die Abstraktion ermöglicht es, die Zusammenhänge im OnlineLernprozess in ihrer wechselseitigen Abhängigkeit zu verstehen.

Die Modelle werden mit der Software Garp3 (Bredeweg et al., 2009) erstellt, einer Workbench, die es ermöglicht, konzeptionelle Ideen mitsamt der Ursache-Wirkungs-Beziehungen zu visualisieren. Mit der Software lassen sich konzeptuelle Modelle über Phänomene darstellen, über die wenige oder keine numerischen Informationen vorliegen. Die Modelle werden nicht etwa mit Zahlen gefüttert, sondern vielmehr mit qualitativen Informationen wie niedrig, hoch, wenige und viele. Allein schon die Formalisierung vorhandenen Domänen-Wissens während der Modellierungsphase kann äußerst wertvoll sein und zu anregenden Diskussionen führen. Beispielsweise könnte man während der Erstellung der konzeptuellen Modelle zu der Erkenntnis gelangen, dass die Experten, die über ein ausgeprägtes Wissen im Bereich des Online-Lernens verfügen (z.B. die

*Kontaktperson: Mag. Christina Schabasser, Am Nasenberg 3, 3133 Traismauer, Österreich, E-Mail: christina.schabasser@live.at Prof. Dr. Bert Bredeweg, University of Amsterdam, Science Park 904 (room C3.109); 1098 XH Amsterdam, Niederlande,

E-Mail: b.bredeweg@uva.nl
Lehrenden von Online-Kursen) ein auffallend unterschiedliches Verständnis vom Online-Lehr-Lernprozess haben. Im Zuge der späteren Analyse der Simulationsergebnisse können neue Perspektiven zur Verbesserung der Qualität des Online-Lernens geschaffen werden. Ausgehend von dem Ziel eines steigenden Lernerfolgs, kann aus den Simulationsergebnissen abgeleitet werden, welche Faktoren dann notwendigerweise auch eine steigende Entwicklung aufweisen müssen bzw. wie die einzelnen Komponenten zusammenspielen müssen, damit der Lernerfolg zumindest durchwegs gleich bleibt.

$\mathrm{Zu}$ Beginn ist es wichtig, sich den gesamten Prozess des Online-Lernens als System mit unterschiedlichen Systemkomponenten vorzustellen, die miteinander in kausaler Beziehung stehen. Um nun die Systemstruktur zu beschreiben, stehen in Garp3 unter anderem sogenannte entities und configurations zur Verfügung. Der Satz „Ein Lehrer interagiert mit einem Schüler" kann durch zwei entities (Lehrer, Schüler) und eine configuration (interagiert) dargestellt werden.

Zur Beschreibung des Systemverhaltens werden quantities, quantity spaces, magnitudes und derivatives, direct influences, proportionalities und inequalities herangezogen. Quantities repräsentieren die Eigenschaften von entities, die sich während einer Simulation verändern und bestehen aus magnitude und derivative. Magnitudes repräsentieren den aktuellen Wert von quantities. Im Gegensatz dazu geben derivatives an, in welche Richtung sich quantities verändern werden. Quantity spaces spielen hierbei eine Rolle, da sie die möglichen Werte angeben, die magnitudes oder derivatives haben können.

Um an das Beispiel oben anzuknüpfen, könnte die entity Schüler durch die quantity Lernaufwand charakterisiert werden. Die quantity Lernaufwand hat den quantity space \{Zero, Plus\}, wobei Zero einen Punktwert und Plus ein Intervall widerspiegelt. Während einer Simulation haben Änderungen von einem Punkt zu einem Intervall immer Vorrang gegenüber Änderungen von einem Intervall zu einem Punkt. Um die Kausalität zwischen quantities auszudrücken, werden positive direct influences oder negative direct influences verwendet. Eine positive direct influence erhöht den derivative von quantity 2, wenn der aktuelle Wert (magnitude) von quantity 1 positiv ist; verringert ihn, wenn der aktuelle Wert von quantity 1 negativ ist und hat keine Aus- 
wirkung auf den derivative von quantity 2, wenn der aktuelle Wert von quantity 1 Null ist. Bei negative direct influences verhält es sich genau umgekehrt.

In Garp3 stehen außerdem positive proportionalities sowie negative proportionalities zur Verfügung, um Kausalität zwischen quantities zum Ausdruck zu bringen. Die positive proportionality erhöht den derivative von quantity 2, wenn der derivative von quantity 1 positiv ist, hat keine Auswirkung, wenn der derivative von quantity 2 stabil ist, und verringert den derivative von quantity 1 , wenn der derivative von quantity 2 negativ ist. Bei einer negative proportionality ist es umgekehrt. Proportionalities bestimmen also derivatives einer quantity 2 auf Grundlage von derivatives einer quantity 1.

Ein Beispiel soll dies verdeutlichen: Um die kausale Beziehung zwischen den quantities Inskription und Anzahl der Teilnehmenden zu beschreiben, könnte eine direct influence verwendet werden. Wenn der aktuelle Wert der quantity Inskription positiv ist, dann erhöht sich der derivative der quantity Teilnehmeranzahl. Inequalities ( $\leq$, $<, 0,>, \geq$ ) bieten eine weitere Möglichkeit zur Beschreibung des Systemverhaltens und werden häufig verwendet, um zu zeigen, dass sich zwei quantity values qualitativ voneinander unterscheiden (Liem et al., 2009).

In der vorliegenden Arbeit finden sich insgesamt vier Modellfragmente, die jeweils einen Teil des Wissens über die Domäne repräsentieren. Ein bestimmtes Szenario gilt dabei als Input für den qualitativen Simulator. Während einer Simulation sucht die Garp3-Engine nach Modellfragmenten, die für das ausgewählte Szenario gelten und leitet das Systemverhalten ab. Die Simulation liefert verschiedene Ergebnisse wie beispielsweise state-graph, value history und equation history. Eine Analyse der Systemstruktur und des Systemverhaltens im Zuge der Interpretation der Simulationsergebnisse kann zu einer neuen Qualität im Online-Lehr-Lernprozess führen.

\section{Die extrinsisch motivierte Teilnahme an Online-Kursen}

Der Lernprozess beginnt mit einer extrinsischen Motivation, einer treibenden Kraft, auf die eine Reaktion folgt. Die Lernmotivation könnte durch den Wunsch ausgelöst werden, den Erwartungen der Eltern zu entsprechen oder Lob vom Lehrer zu bekommen (Lin et al., 2017). Im Beispiel geht der Stimulus von der Marketingbranche aus und bestimmt Wissensniveau und Erwartungen von Unternehmen an seine Mitarbeiter. Das Modellfragment „Auslösetrigger zur Definition des Sollwissens“ (s. Abb. 1) besteht aus den entities „Marketingbranche“ und „Popeye GmbH
Wunschmitarbeiter“, den beiden quantities „Drive“ und „Sollwissen“ sowie der configuration „zaehlt zu“. „Drive“ ist durch den quantity space \{Zero, Plus\} charakterisiert und die quantity „Sollwissen“ hat den quantity space \{Interval\}. Ein positive direct influence (,I+“) beschreibt die Kausalität zwischen „Drive“ und „Sollwissen“. „I+“ bewirkt folgende Entwicklung der quantities: ist der aktuelle Wert von „Drive“ positiv, so steigt „Sollwissen“; ist „Drive“ negativ, sinkt „Sollwissen“; hat die Quantität „Drive“ den Wert 0, dann bleibt „Sollwissen“ unverändert.

Die Erwartung an das Modellfragment lässt sich nun folgendermaßen zusammenfassen: Da das Modellfragment an keine Bedingung geknüpft ist, ist es für die Garp3-Engine im Rahmen der Simulation durchwegs sichtbar und kann angewandt werden. Die Annahme ist, dass „Sollwissen“ der Entwicklung von „Drive“ folgt.

Im Modellfragment „Mechanismus zur Bestimmung des Lernbedarfs“ (s. Abb. 2) wird ein mathematisches Kalkül (Minus) verwendet, um aus der Differenz zwischen „Sollwissen“ und „Istwissen“ die „Rate Wissensbedarf“ zu ermitteln. Die kausale Beziehung zwischen den quantities wird durch eine positive proportionality („P+“") beschrieben, die von der quantity „Sollwissen“ zur quantity „Rate Wissensbedarf“ führt. „P+“ bewirkt, dass „Rate Wissensbedarf“ steigt, wenn „Sollwissen“ steigt, „Rate Wissensbedarf“ abnimmt, wenn „Sollwissen“ abnimmt und „Rate Wissensbedarf“ gleich bleibt, wenn „Sollwissen“ gleich bleibt. Für die negative proportionality (,P-“), so wie sie in der Beziehung zwischen „Istwissen“ und „Rate Wissensbedarf“" verwendet wird, gilt genau das Gegenteil.

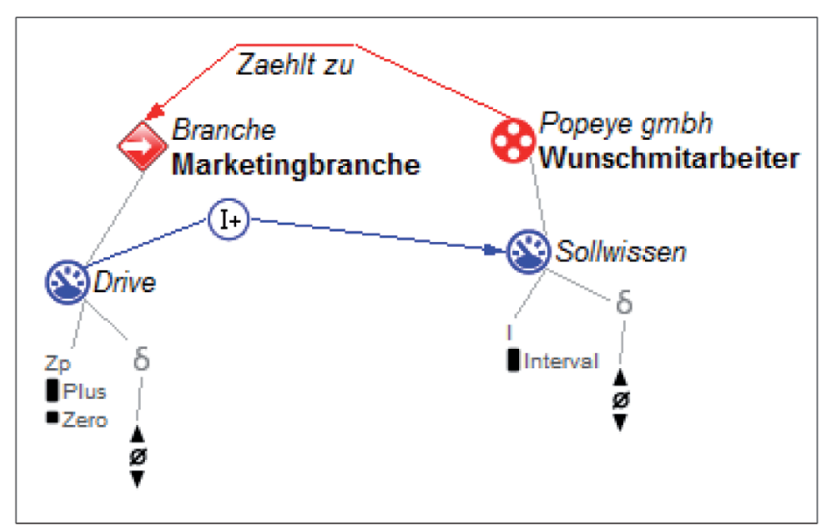

Abbildung 1: Auslösetrigger zur Definition des Sollwissens.

Zur Beschreibung der kausalen Beziehung zwischen „Rate Wissensbedarf“ und „Lernengagement“ wird eine positive proportionality („P+“") verwendet. Die Kausalität zwischen „Lernengagement“ und „Istwissen“ wird durch eine direct influence („I+“) beschrieben. Die Erwartung ist, dass 


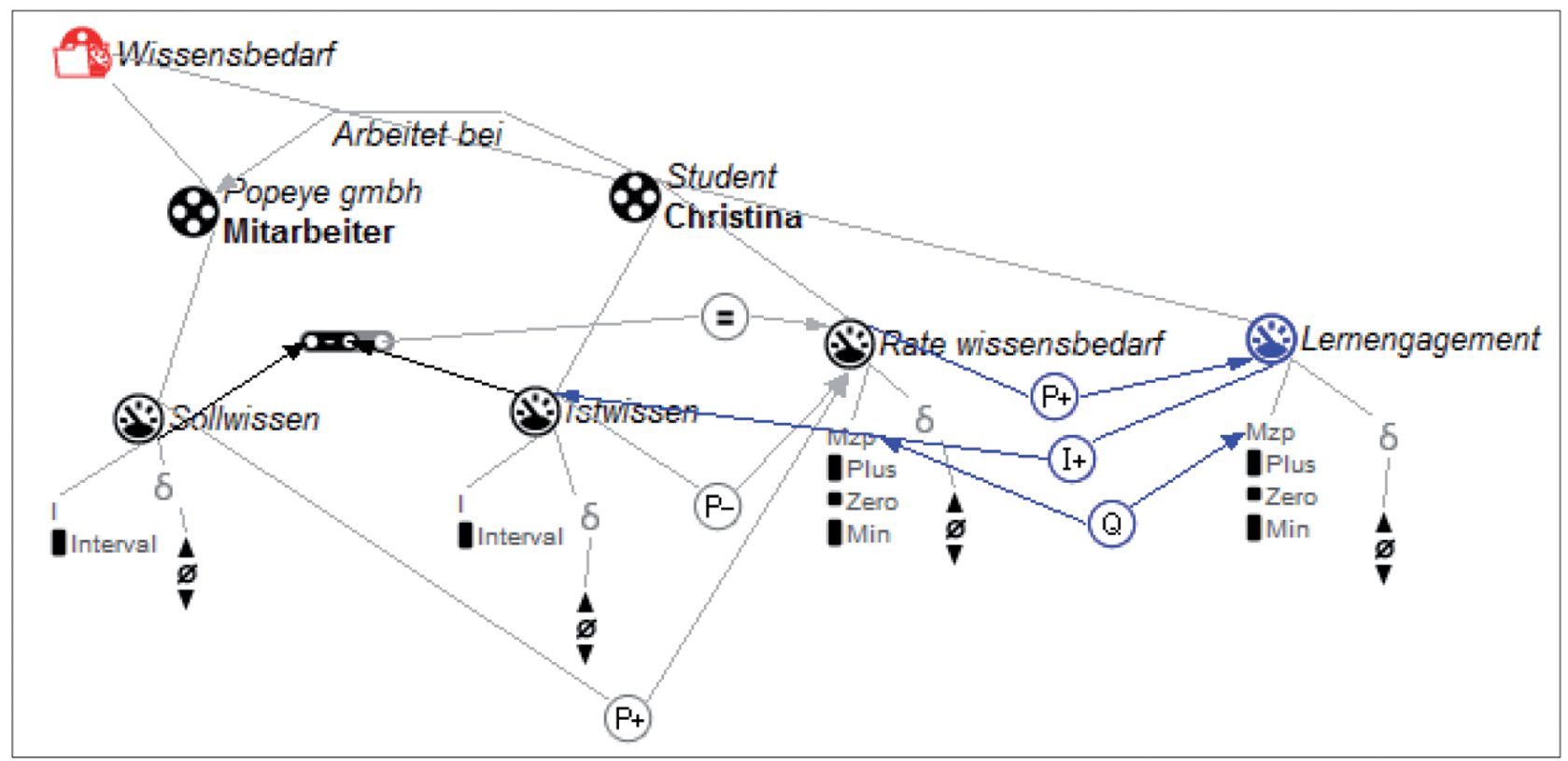

Abbildung 2: Mechanismus zur Bestimmung des Lernbedarfs.

„Lernengagement“ steigt, wenn „Sollwissen“ größer als „Istwissen“ ist. „Lernengagement“ wird solange steigen, bis ein Gleichgewicht (die quantities sind qualitativ gleich) zwischen „Sollwissen“ und Istwissen“ hergestellt ist.

\section{Zur Lerneffektivität in Online-Kursen}

Das virtuelle Lernen innerhalb einer offenen Lernumgebung, also einer Umgebung, in der die Organisation des Lernens durch das lernende Individuum selbst erfolgt, kann als Lernprozess abgebildet werden, der durch Inputund Outputvariablen charakterisiert ist. Es gibt sowohl Inputvariablen des Lernenden (bspw. Motivation) als auch des Lehrenden (z. B. Interventionen). Als Output des Lernprozesses wird der Lernerfolg verstanden. Auf den Lernerfolg selbst wirken Kommunikation und Kooperation mit dem Lehrenden und Qualität und Niveau des Feedbacks ein (Schulmeister, 2004).

Lernengagement und Lernerfolg stehen in Wechselbeziehung. Mit Lernengagement ist die Anstrengung des Lernenden im Lernprozess gemeint, die zu einem bestimmten Lernergebnis führt. Je aktiver sich die Lernenden im Lernprozess engagieren, desto eher können sie ihre Leistungen verbessern. Erfolgreiche Online-Lernende sind motiviert $\mathrm{zu}$ lernen und investieren ausreichend Zeit, um sich auf den Unterricht vorzubereiten (Lee et al., 2019). Neben Vorwissen wird auch die Zeit, die in den Lernprozess investiert wird, als sehr einflussreich auf die Lerneffektivität betrachtet (Ledermüller et al., 2017).

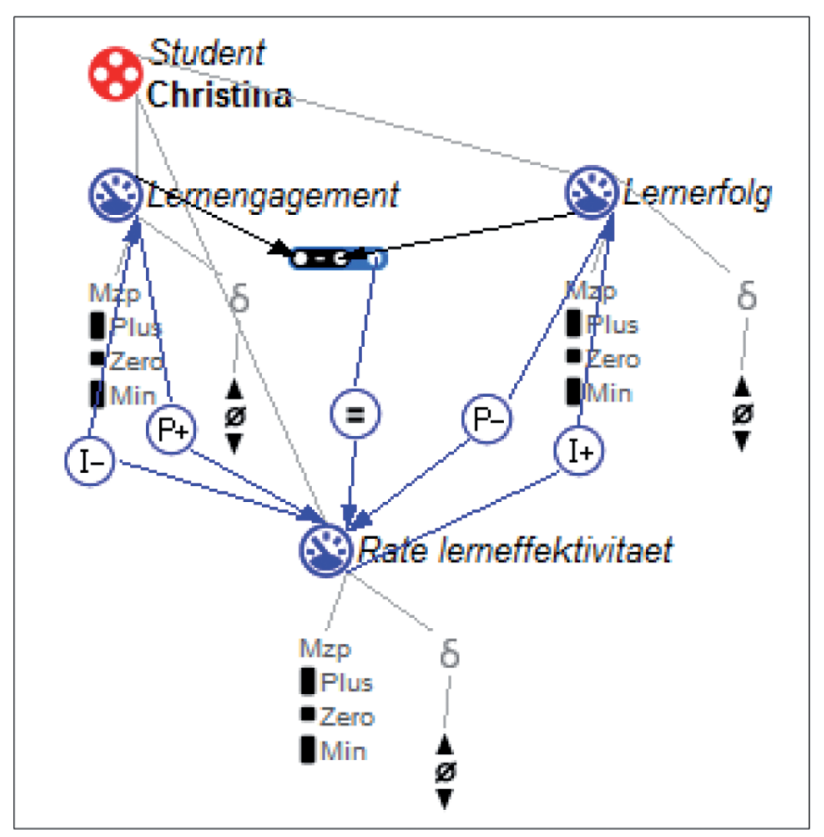

Abbildung 3: Mechanismus zur Lerneffektivität.

Abbildung 3 zeigt das Modellfragment „Mechanismus zur Lerneffektivität“, das aus den quantities „Lernengagement“, „Lernerfolg“ und „Rate Lerneffektivität“ besteht. Im Modellfragment wird das mathematische Kalkül (Minus) verwendet, um aus der Differenz zwischen „Lernengagement“ und „Lernerfolg“ die „Lerneffektivität“ zu ermitteln. Die kausale Beziehung zwischen „Lernengagement“ und „Lernerfolg“ wird durch die negative proportionality „P-“ beschrieben, diese bewirkt, dass „Rate Lerneffektivität“ sinkt, wenn „Lernengagement“ steigt, „Rate 


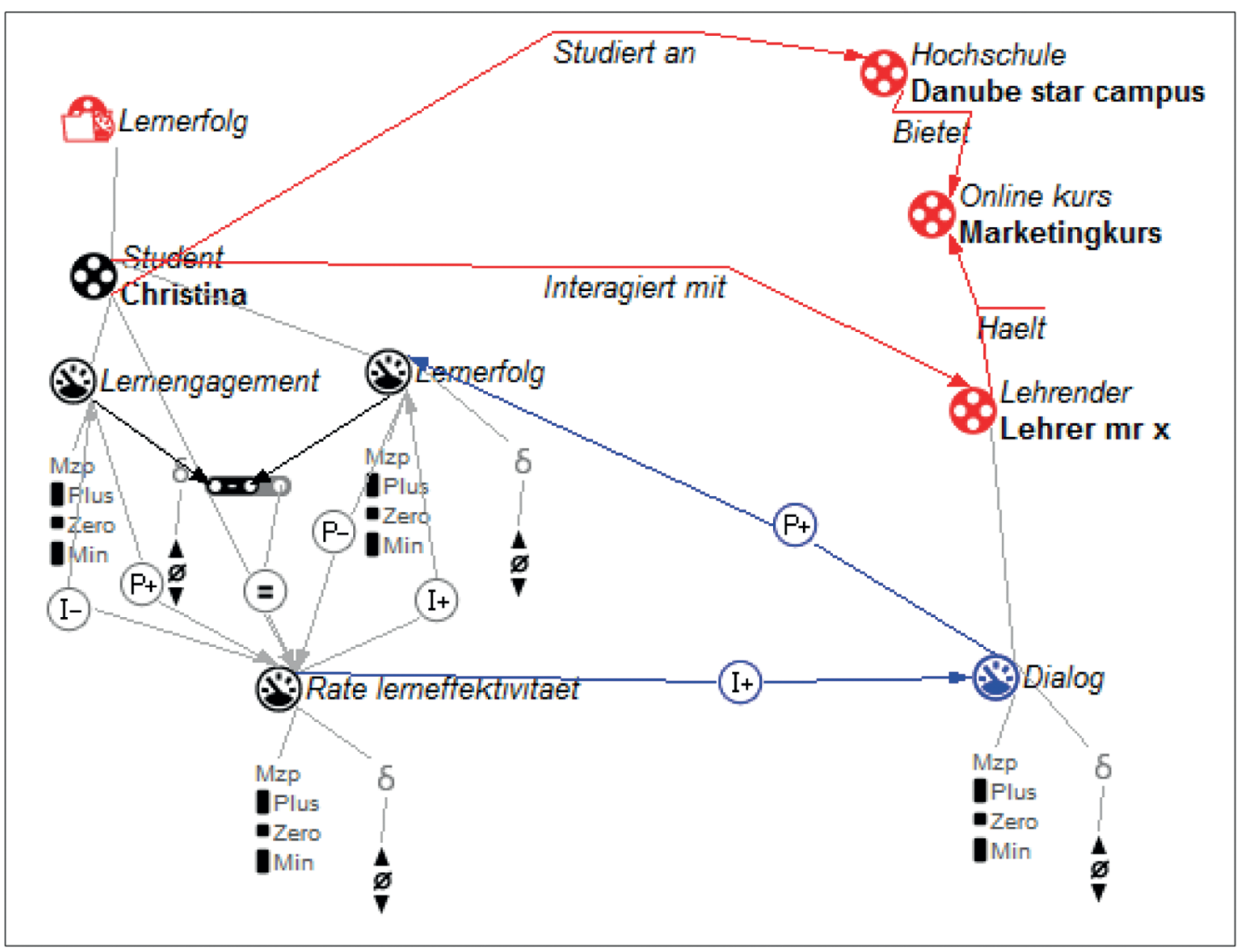

Abbildung 4: Dialog zur Steigerung der Lernperformance.

Lerneffektivität““ steigt, wenn „Lernengagement“ abnimmt und „Rate Lerneffektivität“ gleich bleibt, wenn „Lernengagement“ gleich bleibt. Für die positive proportionality („P +“), so wie sie in der Beziehung zwischen „Lernerfolg“ und „Rate Lerneffektivität“ verwendet wird, gilt genau das Gegenteil. Von „Rate Lerneffektivität“ geht ein e negative influence „I-“ hin $\mathrm{zu}$ „Lernengagement“ und eine positive influence „I+“ hin zu „Lernerfolg“. Die Erwartung ist, dass ein Mechanismus in Gang tritt, wenn sich die beiden quantities „Lernerfolg“ und „Lernengagement“ qualitativ voneinander unterscheiden, also wenn bspw. „Lernengagement“ größer ist als „Lernerfolg“. Dieser Mechanismus soll ein Gleichgewicht herstellen.

\section{Interaktion - entscheidender Faktor für den Lernerfolg}

Die Partizipation von Schülern wird als wesentliche Herausforderung im Bereich des Online-Lernens verstanden. Die Online-Partizipation - und damit ist die Online-Interaktion mit Kommilitonen und Lehrern gemeint - ist eine bedeutende Triebkraft des Lernens. Die Literatur liefert unterschiedliche Betrachtungsweisen der Online-Partizipation von Lernenden. Beispielsweise partizipieren jene Lernende aktiver, die mehr Nachrichten schreiben, häufiger in die Online-Lernumgebung einsteigen oder am Dialog für aktives und engagiertes Lernen teilnehmen (Hrastinkski, 2008; Vonderwell und Zachariah, 2005).

Auch der Begriff Interaktivität muss hier erwähnt werden, dieser ist vom Terminus Interaktion strikt zu trennen und meint im E-Learning-Kontext nicht die soziale Interaktion (Schulmeister, 2004). Mit Interaktivität ist der lernende Umgang mit Lernobjekten wie Fallbeispielen, 


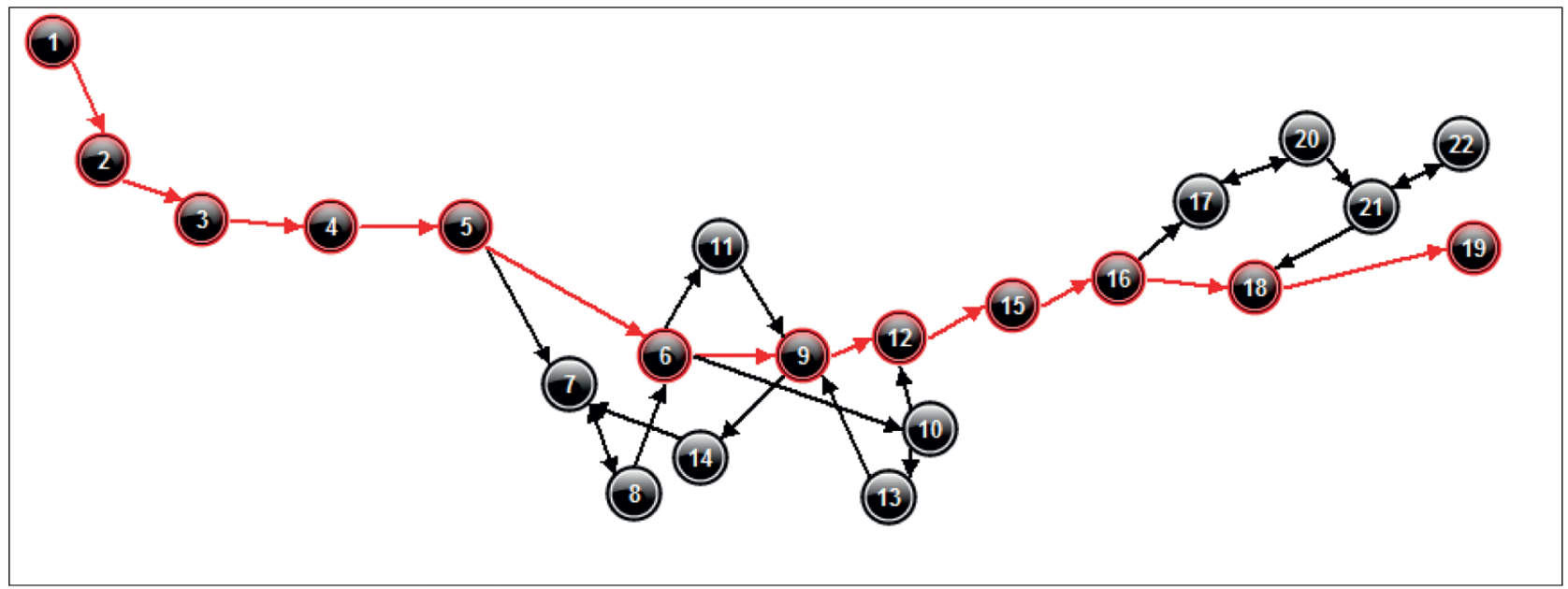

Abbildung 5: state graph mit kürzestem Pfad.

Animationen und Übungen im virtuellen Raum gemeint (Schulmeister, 2003). Der Dialog zwischen Lehrendem und Lernendem ist eine Form der hier erwähnten Interaktion und einer der Makro-Faktoren in der Theorie der transaktionalen Distanz nach Moore. Diese besagt, dass es eine Kommunikationslücke und einen Raum potenzieller Missverständnisse aufgrund der räumlichen Trennung von Lehrendem und Lernendem gibt. Moore beschreibt einen engen Zusammenhang zwischen Struktur und Dialog. Liegt eine geringe Strukturierung und viel Dialog vor, so ist die transaktionale Distanz gering (Moore et al., 1997). Eine möglichst geringe transaktionale Distanz ist wünschenswert.

Im Modellfragment „Dialog zur Steigerung der Lernperformance“ (s. Abb. 4) werden unter anderem die entities „Student Christina“, „Lehrender Lehrer Mr. X“ sowie die Konfiguration „interagiert mit“ eingesetzt, um zu beschreiben, dass es eine Form von Interaktion zwischen Christina und dem Lehrer Mr. X gibt. Wie sich erkennen lässt, wurde das Modellfragment „Mechanismus zur Lerneffektivität“ weiterverwendet und die quantity „Dialog“ wird zur Wiederherstellung eines qualitativen Gleichgewichts zwischen „Lernengagement“ und „Lernerfolg“ eingesetzt, sodass die beiden quantities schließlich den qualitativ gleichen Wert annehmen. Die Erwartung ist, dass der Dialog zwischen Lehrendem und Lernendem steigt, wenn „Lernerfolg“ kleiner als „Lernengagement“ ist.

\section{Diskussion der Simulationsergebnisse}

Die Simulation liefert einen state graph mit 22 Zuständen als Ergebnis (s. Abb. 5), Zustand 19 ist der finale Zustand.
Der kürzeste Pfad $\{1->2->3->4->5->6->9->12->15->16-$ $>18->19\}$ soll näher analysiert werden.

Um Aufschluss über die Entwicklung der einzelnen quantities zu bekommen, wird die value history aus Abbildung 6 herangezogen. „Drive“ steigt in den Zuständen 1, 2, 3 und 4 an, stabilisiert sich in Zustand 5, verzeichnet in den Zuständen 6 und 9 eine rückläufige Entwicklung und behält ab Zustand 15 den qualitativen Wert Zero bei. „Sollwissen“ folgt ab Zustand 2 der Entwicklung von „Drive“, d.h. der qualitative Wert innerhalb des Intervalls steigt. Dieser Anstieg wird bis Zustand 9 fortgesetzt. Die quantity „Sollwissen“ stabilisiert sich ab Zustand 12 und bleibt dann bis zum finalen Zustand unverändert. Um die quantity „Istwissen“ näher zu analysieren, sollte man zeitgleich auch weitere quantities im Blick haben, mit denen die quantity „Istwissen“ eine kausale Beziehung unterhält (bspw. führt eine positive direct influence von „Lernengagement“ zu „Istwissen“).

Ebenso ist es empfehlenswert, einen Blick auf die equation history (s. Abb. 7) zu werfen. Wie die value history aus Abbildung 6 zeigt, steigt „Istwissen“ in den Zuständen 3, 4, 5, 6, 9, 12, 15, 16 und 18. Dieses Verhalten ist damit zu erklären, dass „Istwissen“ genau in den genannten $\mathrm{Zu}$ ständen kleiner als „Sollwissen“ ist (s. Abb. 7) und daher wird hier versucht, ein qualitatives Gleichgewicht zu erreichen bzw. wiederherzustellen (in den Zuständen 1 und 2 war dieses Gleichgewicht vorhanden). Nicht unbeteiligt an diesem Gleichgewicht muss auch die quantity „Lernengagement“ sein. Wenn also „Istwissen“ dazu bewegt werden soll, zu steigen, sollte man auch die Entwicklung von „Lernengagement“ unter die Lupe nehmen, da diese quantity gewissermaßen als Trigger fungiert.

Wie Abbildung 7 zeigt, steigt die quantity „Lernengagement“ ab Zustand 2 (qualitativer Wert Zero), sowie in 


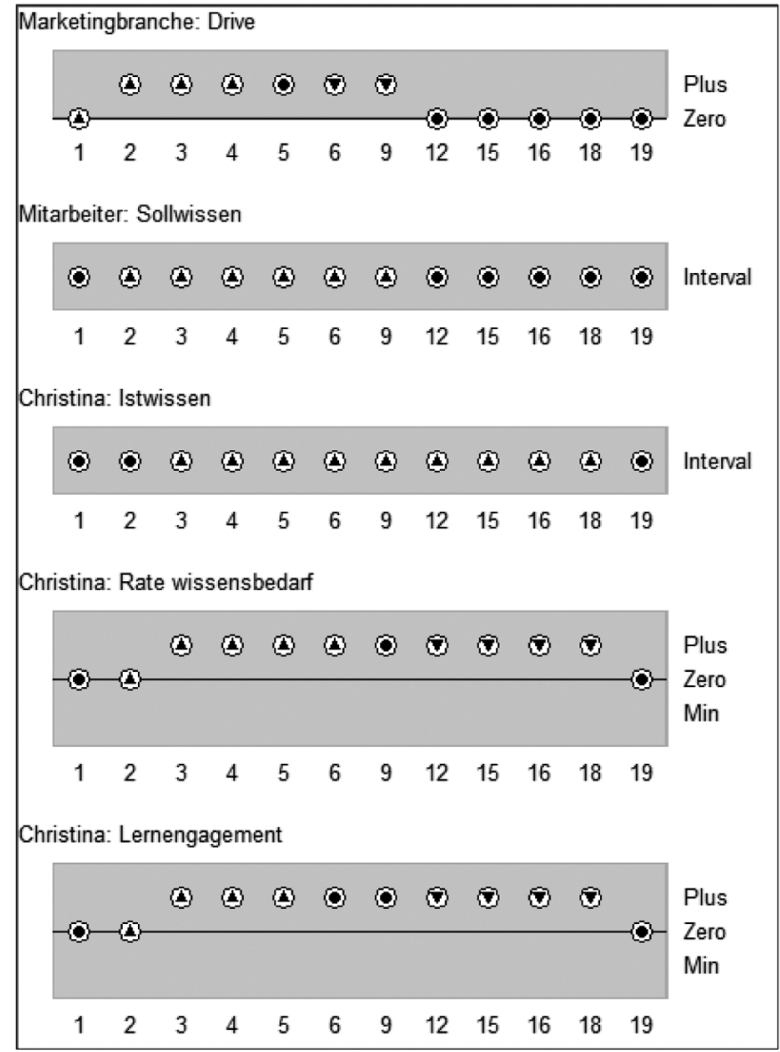

Abbildung 6: value history.

den Zuständen 3, 4 und 5... (qualitativer Wert Plus). In den Zuständen 9 und 12 wird das erste Mal wieder ein Gleichgewicht zwischen „Lernengagement“ und „Lernerfolg“ erreicht (Abb. 7). Die quantity „Dialog“ steigt ab Zustand 3 (qualitativer Wert Zero) und setzt die steigende Entwicklung bis Zustand 6 fort (qualitativer Wert Plus). In den Zuständen 9 und 12 stabilisiert sie sich im Intervall Plus, wo sie den qualitativen Wert beibehält. Ab Zustand 15 sinkt der qualitative Wert der quantity „Dialog“.



Abbildung 7: equation history.

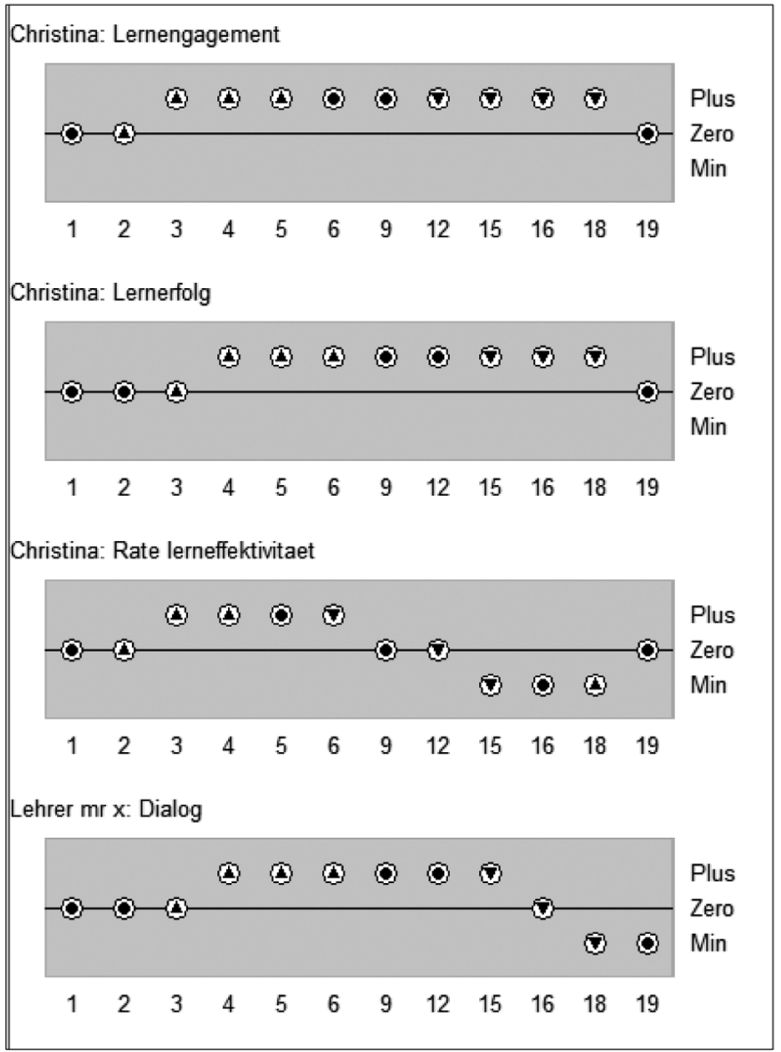

\section{Diskussion und Ausblick}

Das Angebot an Online-Kursen nimmt stetig zu. Um das Online-Lehrangebot optimal zu gestalten und fortlaufend $\mathrm{zu}$ verbessern, ist es wichtig, die kausalen Zusammenhänge zu kennen, die einen Online-Lehr-Lernprozess charakterisieren. Die Software Garp3 bietet die Möglichkeit, domänenspezifisches Wissen über webbasierte Pädagogik in Form von konzeptuellen Modellen abzubilden. Schon die Formalisierung des Domänenwissens stellt sich hier als große Bereicherung heraus, da sie zwingt, sich Gedanken über Ursache-Wirkungs-Zusammenhänge $\mathrm{zu}$ machen.

Beim Aufarbeiten relevanter Literatur fällt auf, dass es Unterschiede in der Benennung verschiedenster Phänomene beim Online-Lernen gibt. Beispielsweise ist nicht immer von Lernengagement die Rede, sondern oftmals wird der Fokus auf die investierte Zeit gelegt oder es wird der Begriff Lernaufwand verwendet. Unter diesem Gesichtspunkt liefern die Modelle viel Raum für anregende Diskussionen. Würde derjenige, der das Modell liest und interpretiert, dasselbe Wording verwenden, um bestimmte Phänomene zu beschreiben? Oder eröffnen ihm die konzeptuellen Modelle etwa ganz neue Perspektiven? 
In seiner verantwortungsvollen Rolle verfolgt der Online-Lehrende das Ziel, den Lernerfolg im Online-Kurs zu optimieren. Welche Hebel existieren zur Erreichung dieses Ziels? Ist es ausreichend, wenn das Lernengagement des Lernenden vorhanden ist, allerdings keine oder kaum Interaktion zwischen Lernendem und Lehrendem stattfindet? Wann sollte sich der Lehrende mehr Zeit für Interaktion und Dialog nehmen? Gerade der Zeitpunkt von pädagogischen Interventionen kann sich als bedeutsam herausstellen, so beispielsweise beim Erteilen von Feedback, da man dieses besser annimmt, wenn es zeitnah gegeben wird. Wenn es um pädagogische Interventionen geht, ist es wichtig, die Ressourcen im Fokus zu haben. Die Simulationsergebnisse lassen unter anderem die Interpretation $\mathrm{zu}$, dass Interventionen (bspw. verstärkter Dialog zwischen Lehrendem und Lernendem) nur in bestimmten Fällen verstärkt notwendig sind, z. B. wenn sich der Lernerfolg trotz hohem Lernengagement nicht einstellen will.

Die vorgestellten Modelle erheben keinen Anspruch auf Vollständigkeit. Vielmehr soll eine neue Art des Denkens und des Erfassens von Domänenwissen über OnlineKurse präsentiert und erste Simulationsergebnisse diskutiert werden. In der aktuellen Forschung werden die Modelle um weiteres Domänenwissen ergänzt. Konkret werden die Modelle um die quantities „Struktur“ und „Autonomie“ erweitert, um die Theorie der transaktionalen Distanz gebührend einzubeziehen.

Deskriptoren: Modell, Online, Lernen, Online-Kurse, E-Learning, Lernprozess, Konzeptionelle Modellierung, Garp3

\section{Literatur}

Bredeweg, Bert; Linnebank, Floris; Bouwer, Anders; Liem, Jochem, 2009. Garp3 - Workbench for qualitative modelling and simulation. In: Ecological Informatics. 4 (5-6), S. 263-281. ISSN: 1574-9541.

Hrastinski, Stefan, 2008. What is learner participation? A literature review. In: Computer \& Education. 51 (4), S. 1755-1765. ISSN: 0360-1315.

Ledermüller, Karl and Fallmann, Irmgard, 2017. Predicting learning success in online learning environments: Self-regulated learning, prior knowledge and repetition. In: Zeitschrift für Hochschulentwicklung, 12 (1). S. 79-99. ISSN 22196994.

Lee, Jeongju; Song, Hae-Deok; Hong, Ah Jeong, 2019. Exploring Factors, and Indicators for Measuring Students' Sustainable Engagement in e-Learning. In: Sustainability. 11 (4).

S. 985-997. ISSN: 2071-1050.

Liem, Jochem; Linnebank, Floris; Bredeweg, Bert, 2009. Model Building Experiences using Garp3: Problems, Patterns and Debug- ging. In: 23rd International Workshop on Qualitative Reasoning (QR'09), Ljubljana, Slovenia. Conference Paper.

Lin, Ming-Hung; Chen, Huang-Cheng; Liu, Kuang-Sheng, 2017. A Study of the Effects of Digital Learning on Learning Motivation and Learning Outcome. In: Eurasia Journal of Mathematics, Science and Technology Education, 13(7), S. 3553-3564. https:// doi.org/10.12973/eurasia.2017.00744a.

Moore, Michael, 1997. Theory of transactional distance. In: Keegan, D. (Hg.): Theoretical Principles of Distance Education, Routledge, S. 22-38.

Schulmeister, Rolf, 2003. Lernplattformen für das virtuelle Lernen. München, Wien: Oldenbourg. ISBN: 3486272500.

Schulmeister, Rolf, 2004. Didaktisches Design aus hochschuldidaktischer Sicht. Ein Plädoyer für offene Lernsituationen. In: U. Rinn \& D. M. Meister (Hrsg.), Didaktik und neue Medien. Konzepte und Anwendungen in der Hochschule (S. 19-49). Münster; New York: Waxmann. ISBN: 3830912161.

Vonderwell, Selma; Zachariah, Sajit, 2005. Factors that Influence Participation In Online Learning. In: Journal of Research on Technology in Education. 38 (2), S. 213-230. ISSN: 1945-0818.

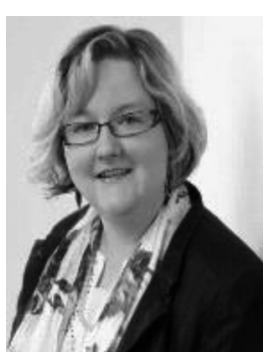

Mag. Christina Schabasser Am Nasenberg 3

3133 Traismauer

Österreich

christina.schabasser@live.at

Christina Schabasser studierte Internationale Betriebswirtschaft an der Universität Wien. Nach ihrem Studium war sie viele Jahre in der Softwarebranche tätig. Derzeit arbeitet sie als Redakteurin und Channel Managerin in Wien.

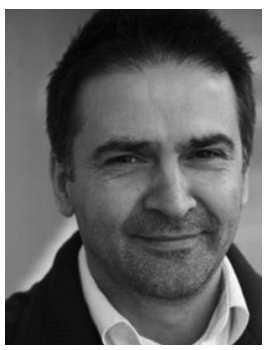

\section{Prof. Dr. Bert Bredeweg}

University of Amsterdam

Science Park 904 (room C3.109)

1098 XH Amsterdam

Niederlande

b.bredeweg@uva.nl

Bert Bredeweg ist außerordentlicher Professor am Informatik-Institut der Universität Amsterdam und leitet die Qualitative Reasoning Group. Sein Forschungsschwerpunkt liegt in der Entwicklung von Werkzeugen und Know-how, das dem Erwerb von konzeptuellem Verständnis dient. 